\title{
Espectrômetro digital. Uma proposta de construção de um experimento de Física Moderna para o ensino remoto
}

\author{
Digital spectrometer. A proposal to build a Modern Physics experiment for remote learning \\ Espectrómetro digital. Una propuesta para construir un experimento de Física Moderna para el \\ aprendizaje remoto
}

Recebido: 01/07/2021 | Revisado: 06/07/2021 | Aceito: 06/07/2021 | Publicado: 17/07/2021

\author{
Jaykel Joana de Jesus \\ ORCID: https://orcid.org/0000-0002-6620-9347 \\ Universidade Federal de Itajubá, Brasil \\ E-mail: jayke198@unifei.edu.br \\ Adhimar Flávio de Oliveira \\ ORCID: https://orcid.org/0000-0003-2586-7359 \\ Universidade Federal de Itajubá, Brasil \\ E-mail: adhimarflavio@unifei.edu.br \\ Agenor Pina da Silva \\ ORCID: https://orcid.org/0000-0002-1445-1027 \\ Universidade Federal de Itajubá, Brasil \\ E-mail: agenor@unifei.edu.br
}

\begin{abstract}
Resumo
Com o surgimento da pandemia de Covid 19, o ensino sofreu um choque muito grande, que fez com que os docentes buscassem nas tecnologias soluções para o ensino. As tecnologias passaram a ser considerada como a única opção para se ensinar em tempos de reclusão e passaram a estar mais presentes no cotidiano do professor, principalmente para substituir as atividades experimentais desenvolvidas presencialmente no ensino superior ou para proporcionar atividades remotas para o ensino básico. Neste contexto, apresenta-se neste trabalho as etapas de construção de um espectrômetro digital a partir de materiais reaproveitados de sucata de equipamentos eletrônicos, câmera CCD e mídias como CDs ou DVDs usados, além do software Theremino que é gratuito para aquisição de dados, visualização em tempo real e análise posterior. O experimento construído foi concebido com o objetivo de utilização em aulas experimentais no formato remoto e ou presencial em atividades multidisciplinares que envolvam o estudo do espectro eletromagnético de diferentes fontes de radiação e os fenômenos da interação com a matéria. O espectro eletromagnético traz inúmeras informações em pesquisas nas áreas de astronomia, física, química e biologia. Seu estudo também é muito significativo no ensino de física e astronomia, pois permitindo discutir diversos fenômenos físicos do cotidiano dos alunos, que envolvem o comportamento da radiação em diferentes comprimentos de ondas. O espectrômetro digital apresentado é um equipamento de baixo custo é uma ferramenta interessante para auxiliar os alunos a entenderem o comportamento da radiação eletromagnética em diferentes comprimentos de onda e a importância da radiação eletromagnética em aplicações no cotidiano, tais como, a necessidade de utilização de filtro solar até fenômenos tecnológicos como o efeito fotoelétrico na interação da radiação com dispositivos semicondutores.
\end{abstract}

Palavras-chave: Espectro eletromagnético; Espectrômetro digital; Experimentação remota; Experimentação de baixo custo; Ensino remoto; Ensino.

\begin{abstract}
With the emergence of the Covid 19 pandemic, teaching suffered a very big shock, which made teachers look to technology for solutions for teaching. Technologies started to be considered as the only option to teach in times of confinement and started to be more present in the daily life of the teacher, mainly to replace the experimental activities developed in-person in higher education or to provide remote activities for basic education. In this context, this work presents the stages of construction of a digital spectrometer from reused materials from electronic equipment scrap, CCD camera, and media such as used CDs or DVDs, in addition to the Theremino software, which is free for data acquisition, visualization real-time and post-analysis. The experiment built was conceived to use in experimental classes in remote and/or in-person format in multidisciplinary activities that involve the study of the electromagnetic spectrum of different radiation sources and the phenomena of interaction with matter. The electromagnetic spectrum provides a wealth of information in research in the fields of astronomy, physics, chemistry, and biology. Its study is also very significant in the teaching of physics and astronomy, as it allows discussing various physical phenomena in the daily lives of students, which involve the behavior of radiation at different wavelengths. The digital spectrometer presented
\end{abstract}


is low-cost equipment and an interesting tool to help students understand the behavior of electromagnetic radiation at different wavelengths and the importance of electromagnetic radiation in everyday applications, such as the need to use a filter to technological phenomena such as the photoelectric effect in the interaction of radiation with semiconductor devices.

Keywords: Electromagnetic spectrum; Digital spectrometer; Remote experimentation; Low-cost experimentation; Remote learning; Teaching.

\section{Resumen}

Con la aparición de la pandemia de Covid 19, la enseñanza sufrió un gran impacto, lo que hizo que los profesores buscaran soluciones para la enseñanza en la tecnología. Las tecnologías comenzaron a ser consideradas como la única opción para enseñar en tiempos de encierro y comenzaron a estar más presentes en la vida diaria del docente, principalmente para reemplazar las actividades experimentales desarrolladas presencialmente en la educación superior o para brindar actividades a distancia para la educación básica. . En este contexto, este trabajo presenta las etapas de construcción de un espectrómetro digital a partir de materiales reutilizados de desechos de equipos electrónicos, cámara CCD y medios como CD o DVD usados, además del software Theremino, que es gratuito para adquisición de datos, visualización. en tiempo real y posanálisis. El experimento construido fue concebido para ser utilizado en clases experimentales en formato remoto y / o presencial en actividades multidisciplinares que involucran el estudio del espectro electromagnético de diferentes fuentes de radiación y los fenómenos de interacción con la materia. El espectro electromagnético proporciona una gran cantidad de información en la investigación en los campos de la astronomía, la física, la química y la biología. Su estudio es también muy significativo en la enseñanza de la física y la astronomía, ya que permite discutir diversos fenómenos físicos en la vida diaria de los estudiantes, que involucran el comportamiento de la radiación en diferentes longitudes de onda. El espectrómetro digital presentado es un equipo de bajo costo y una herramienta interesante para ayudar a los estudiantes a comprender el comportamiento de la radiación electromagnética en diferentes longitudes de onda y la importancia de la radiación electromagnética en aplicaciones cotidianas, como la necesidad de utilizar un filtro para fenómenos tecnológicos como el fotoeléctrico. efecto en la interacción de la radiación con dispositivos semiconductores.

Palabras clave: Espectro electromagnético; Espectrómetro digital; Experimentación remota; Experimentación de bajo costo; Aprendizaje remoto; Enseñanza.

\section{Introdução}

O uso de espectroscópico de baixo custo como uma estratégia para o ensino do espectro eletromagnético vem sendo apresentado em diversos trabalhos relacionados ao ensino de Física. Nesses trabalhos, segundo seus autores, o espectrógrafo foi utilizado como uma alternativa para o ensino de Astronomia (Barros, Assis e Langhi, 2016), em outros ele é utilizado para o ensino de Física Quântica (Azevedo, Sousa e Castro, 2019; Santana e Santos, 2017) ou utilizados para o ensino da óptica física (Bruzadin, 2018).

Mesmo este tipo de trabalho ter sido bastante explorado na literatura, atualmente sugiram propostas que associam o uso desses espectrógrafos com recursos tecnológicos para auxiliar o professor no processo de ensino e aprendizagem no ensino de física. De acordo com Silva, Tavares e Silva (2018, p.2), o professor nos dias de hoje

possui à sua disposição uma infinidade de recursos tecnológicos que possam servir como meios auxiliares no processo construção do conhecimento. Atualmente, o computador se apresenta como o grande aliado, ao passo que é possível operar programas específicos para cada área do conhecimento ou até mesmo softwares para cada assunto de interesse.

Apesar de todos esses recursos disponíveis, a utilização das tecnologias no ambiente escolar ainda não é uma realidade em muitas instituições de ensino brasileiras. A falta de estrutura de nossas escolas e a formação deficitária do professor, por exemplo, podem ser apontadas como duas dificuldades para a inserção tecnológica na sala de aula e ao envolvimento dos professores na utilização dessa metodologia em sala de aula. Esta realidade é responsável por uma formação científica e tecnológica bastante deficiente aos estudantes das escolas brasileiras, deixando-os cada vez mais defasados em relação ao cenário de desenvolvimento tecnológico mundial.

Entretanto, esta realidade, especialmente no contexto escolar, sofreu um choque muito intenso com o surgimento da pandemia de Covid 19 (Schuchat et al. 2020; Guenther, 2020), que fez (e ainda faz) com que os docentes busquem nas tecnologias 
soluções tecnológicas para o ensino (Barin et al. 2021; dos Santos et al. 2020). As tecnologias passaram a ser considerada como a única opção para se ensinar em tempos de reclusão e passaram a estar mais presentes no cotidiano do professor, principalmente para substituir as atividades experimentais desenvolvidas presencialmente no ensino superior ou para proporcionar atividades remotas para o ensino básico, posto que laboratórios de física de qualidade são praticamente inexistentes na maioria das escolas de ensino público e privado da educação básica.

É neste contexto que este trabalho está inserido, isto é, a de propor um experimento voltado ao estudo do espectro eletromagnético e que está diretamente relacionado com o uso do computador, seguindo as metodologias apresentadas em Pereira et.al. (2018). Trabalhos deste tipo tem sido discutido por Perkampusmar (2013) e Azevedo et al. (2019). A proposta deste trabalho reside em apresentar o desenvolvimento de uma atividade experimental relacionada ao espectro eletromagnético que envolve a construção de um espectroscópio de baixo custo e a utilização do software Theremino, um software gratuito que permite a aquisição de dados, a visualização em tempo real e a análise de dados. $\mathrm{O}$ experimento apresentado aqui foi desenvolvido com o objetivo de ser utilizado de forma remota e ou presencial em atividades multidisciplinares que envolvam o estudo do espectro eletromagnético de diferentes fontes de radiação e o fenômeno de absorção da radiação na passagem por diferentes tipos de materiais Toledo et al. (2020). O espectrômetro digital apresentado é uma ferramenta interessante para auxiliar os alunos a entenderem o comportamento da radiação eletromagnética em diferentes comprimentos de onda na região do visível e próxima ao visível e sua importância em atividades cotidianas, tais como, a simples utilização de filtro solar e óculos escuros. Além disso, este experimento pode ser construído e realizado de forma síncrona ou assíncrona pelo docente.

\section{Revisão Teórica}

De acordo com o modelo de Bohr, para ocorrer a absorção ou emissão de radiação pelo átomo, o elétron precisa transitar entre estados de energia diferentes. A radiação emitida ou absorvida está relacionada com a mudança de nível, quando o elétron muda de nível de excitação mais alto para um inferior, ocorre a emissão de um fóton. Na Figura 1 é mostrada uma ilustração do átomo de Bohr e algumas transições entre níveis de energia desses átomos. A frequência em que os fótons emitidos serão detectados dependo da energia desses fótons e, neste experimento, será utilizado um espectrômetro para esse fim.

Figura 1: Ilustração do modelo de Bohr para o átomo, com as transições entre os níveis de energia. a) Cada órbita corresponde a um nível de energia. Quanto mais afastado do núcleo, mais energia tem o elétron. b) Quando o elétron recebe energia, ele salta do primeiro nível a um nível superior, distanciando-se do núcleo. c) Quando o elétron salta de um nível até o outro mais próximo ao núcleo, ele libera energia. Essa liberação pode ocorrer na forma de luz.

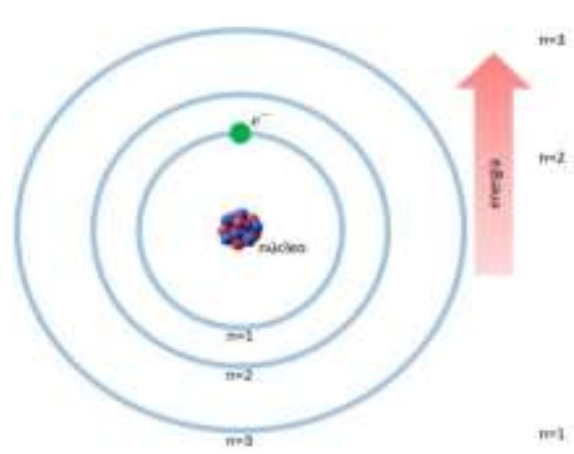

$m$

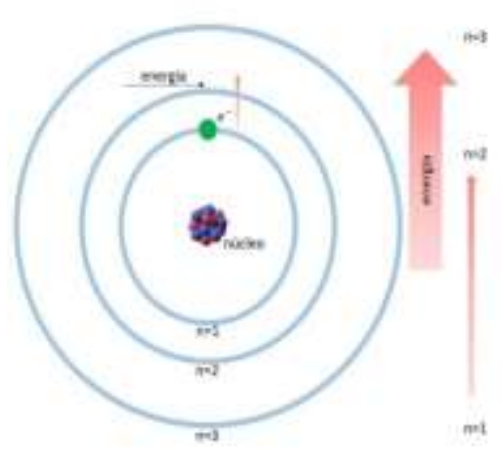

Fonte: Autores. 
O espectro eletromagnético é a divisão em todas as frequências, ou comprimentos de onda, possível para a radiação eletromagnética. Como pode ser verificado na Figura 2, o espectro eletromagnético cobre desde ondas de rádio, em baixas frequências e energia, até a radiação Gama em grandes frequências e energias.

Com a evolução das técnicas e dos instrumentos para se observar a radiação eletromagnética ao longo do espectro eletromagnético, foi possível obter informações de diferentes processos físicos responsáveis pela emissão da radiação eletromagnética, desde os fenômenos menos energéticos (detectados nas ondas de rádio, nas micro-ondas, infravermelho, ultravioleta) até os eventos mais energéticos do universo (detectados em raios X e raios gama). A observação da radiação visível e regiões do Infravermelho (IV), faixas de interesse neste trabalho, podem ser observadas através de espectrômetros. A espectroscopia se transformou numa ferramenta muito importante na analise de como a matéria interage em relação à absorção ou emissão de luz.

Figura 2: Divisão do espectro eletromagnético em diferentes comprimentos de onda e o valor equivalente para frequências. Em evidência é apresentada a faixa de radiação visível.

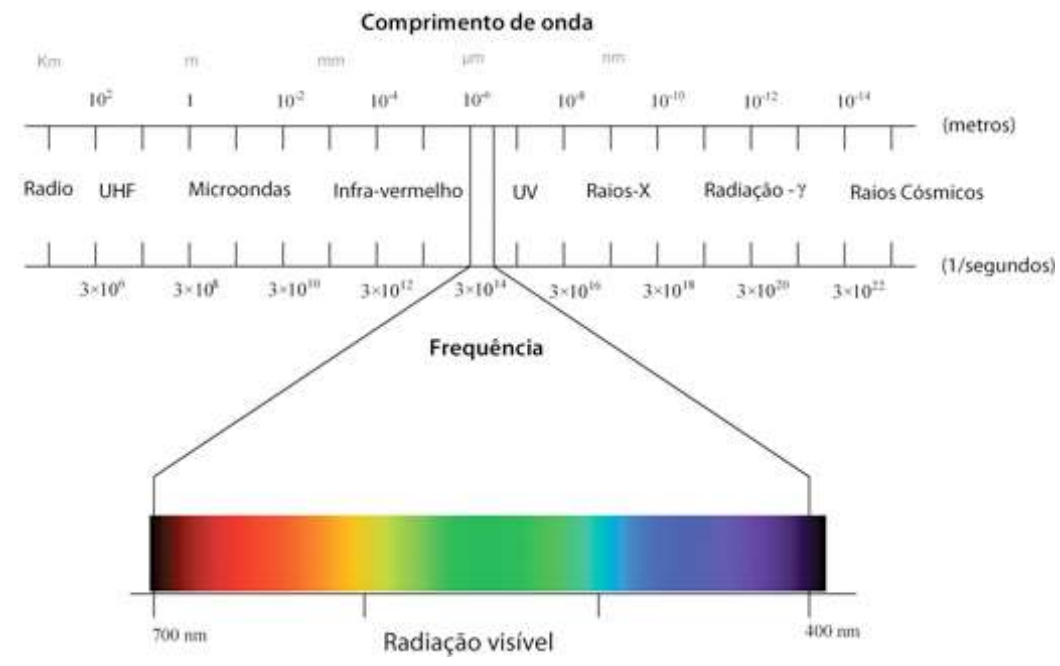

Fonte: Rodrigues \& Brizola (2019).

Em 1860 Kirchhoff formulou as leis que apresentam os três tipos de espectros existentes. Esses espectros estão mostrados na Figura 3: um corpo opaco quente, sólido, líquido ou gasoso, emite um espectro contínuo; um gás transparente produz um espectro de linhas brilhantes (de emissão); e se um espectro contínuo passar por um gás à temperatura mais baixa, o gás frio causa a presença de linhas escuras (absorção). O número e a posição das linhas dependem dos elementos químicos presentes no gás. 
Figura 3: Gráfico representando os três tipos de espectros possíveis formulado por Kirchhoff.

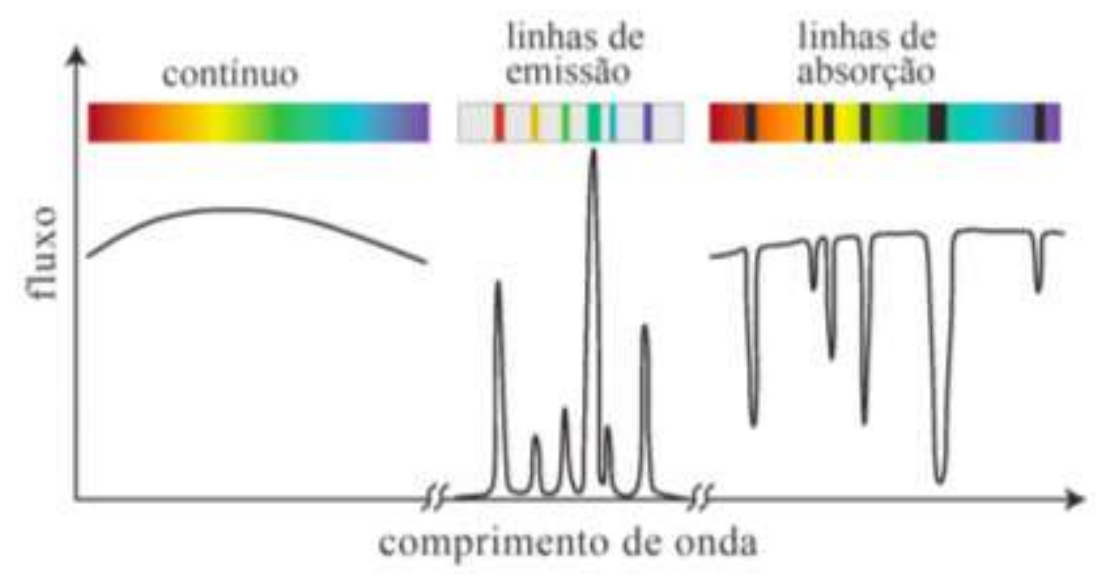

Fonte: http://lilith.fisica.ufmg.br/ guerrero/notas-fis004/05-Distribuicao-Energia-Linhas-Espectrais.pdf

O resumo teórico apresentado nesta seção foi colocado pois esses pontos podem ser trabalhados na atividade experimental que está sendo proposta neste trabalho. Não foi nossa intenção discutir esses pontos profundamente. Entendemos que eles são apresentados em disciplinas de física do ensino superior e que também podem ser tratados no terceiro ano do ensino médio.

\section{Metodologia}

Como já estabelecido em diversos trabalhos apresentados na literatura que propõem a construção de espectroscópios de baixo custo, neste trabalho também foi utilizado um CD para a obtenção de uma rede de difração, que é um dispositivo com um grande número de linhas, ou fenda, igualmente espaçadas em uma superfície plana. Dependendo do modo como é produzida, esta rede pode difratar a luz tanto por reflexão quanto por transmissão. Em nosso caso ela será difratada por transmissão. O modo de construção desta rede a partir do CD está ilustrado na Figura 4. A película refletora da mídia é removida, uma dica é utilizar o auxílio de um estilete e uma fita isolante, como mostrado nas Figuras 4(a) e 4(b). Em seguida, cortou-se um pedaço radial da mídia e foi encaixada em um pedaço quadrado de um papelão, sendo o pedaço da mídia encaixada em um rasgo e fixada com fita isolante, como apresentado na Figura 4(c).

Figura 4: Montagem da rede de difração. Em (a) é apresentada a etapa de remoção da película refletora, (b) o corte radial da mídia e (c) a rede de difração montada com o papelão e a fita isolante.

(a)

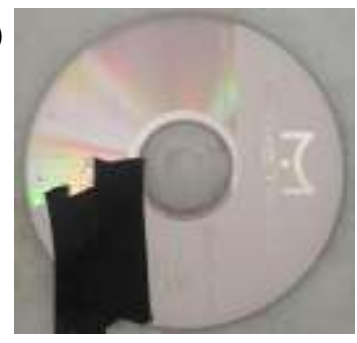

(b)

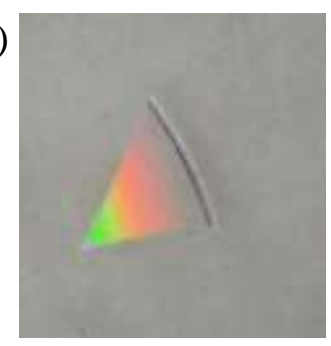

Fonte: Autores. (c)

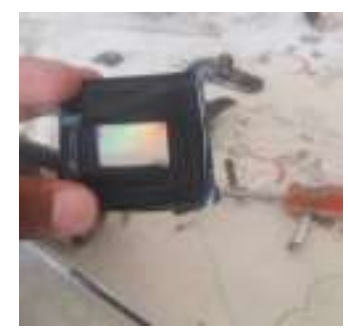

Foi necessário fazer a conversão da radiação difratada pela rede de difração para a forma digital. Para isso, foi utilizada uma câmera de dispositivo de carga acoplada (do inglês charge-coupled device, CCD) retirada de uma webcam. Após atravessar 
a rede de difração, a mesma é capturada pelo CCD e enviada ao software, via cabo USB, e seu espectro eletromagnético é projetado na tela do computador, ajudando na diferenciação dos diferentes comprimentos de onda e na análise posterior dos dados.

Uma das etapas da montagem é feita adaptando a câmera CCD para a leitura de uma faixa maior espectro eletromagnético. Para medir a luz infravermelha (IR) é preciso retirar o filtro IR na parte posterior da webcam. Nem todas as câmeras webcam possuem este filtro, mas é bem fácil e simples de verificar se a câmera possui ou não, é só desrosquear a parte frontal da câmera e verificar a existência do filtro. Na Figura 5 é mostrado como foi realizada a operação de remoção do filtro IR.

Figura 5: Remoção do filtro IR da webcam. Esta etapa é importante para aumentar a faixa de comprimentos de onda observadas no espectrômetro digital.

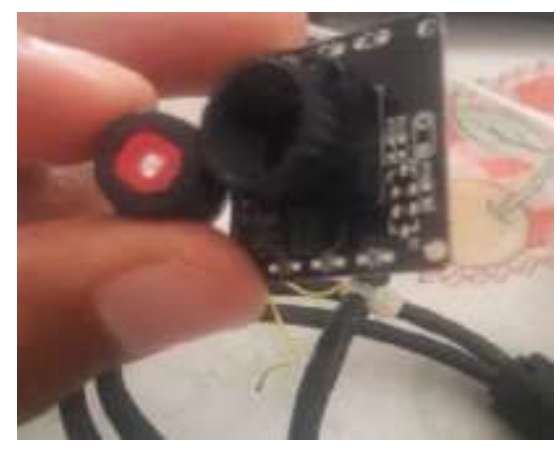

Fonte: Autores.

Para maior estabilidade do sistema óptico foi necessário colocar a câmera CCD dentro de um suporte. Para isso, foi utilizado um rolo de papel higiênico e a câmera foi fixada em cima de pedaços retangulares do papelão com cola quente, Figura 6 (a). Depois de todo o procedimento, foi fixada a rede de difração na câmera para colocar dentro da caixa, como apresentado nas Figuras 6 (b) e 6 (c).

Figura 6: (a) Fixação da câmera CCD em um rolo de papel para estabilizar o sistema, (b) fixação da rede de difração na câmera e (c) finalização da câmera CCD.

(a)

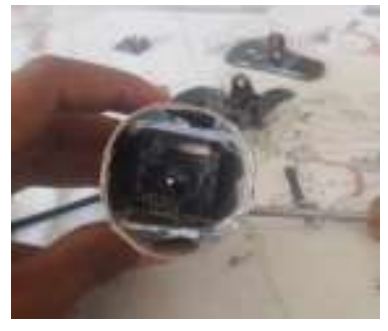

(b)

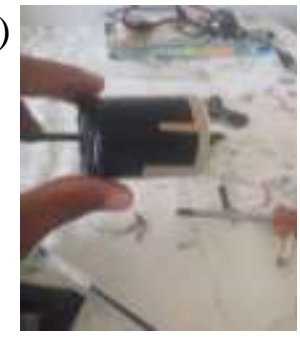

Fonte: Autores.

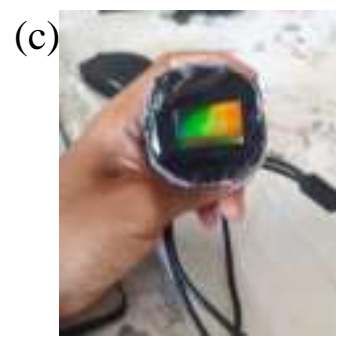

O espectrômetro foi acondicionado em uma caixa de papelão pintada na cor preta para reduzir ao máximo a interferência por reflexão nas paredes da caixa. Para isso, foi necessário fazer uma entrada na tampa da frente para a passagem da luz. Junto à entrada foi fixada uma fenda feita de lâminas de barbear para fazer com que a luz incidente passe por uma abertura mais estreita e assim obter feixe de ondas mais definidos quando a luz sofrer difração ao incidir no CD. Outra entrada foi feita no fundo da caixa para a passagem do cabo USB da câmera, e para fixar a tampa foi utilizada fita isolante vedando a passagem de luz. Para 
ajudar na compreensão da montagem, apresentamos na Figura 7 as etapas de preparação da caixa.

Figura 7: (a) Preparação da caixa para acondicionamento e (b) fixação da fenda feita através de lâminas de barbear.

(a)

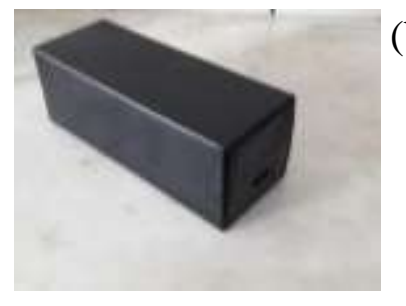

(b)

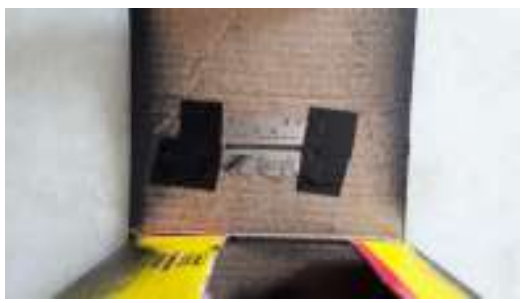

Fonte: Autores.

Para a coleta de dados optou-se pela utilização do software Theremino (Theremino, 2021). O Theremino é um sistema Open Source que pode ser utilizado em diferentes funcionalidades, como, por exemplo, nas músicas, no ensino e pesquisa científica, como pode ser verificado em sua página na internet (Theremino, 2021). Ao contrário de sistemas semelhantes (por exemplo, Arduino), o sistema funciona assim que é ligado e não requer programação de firmware. Na Figura 8 é apresentada a tela do software Theremino operando para análise de um espectro eletromagnético. No retângulo menor da cor de fundo preto, no qual está a leitura da webcam, o parâmetro 'Size Y' representa o número de linhas de pixels usados para a análise. O software faz a média dos valores de todas as linhas e isso melhora a sensibilidade e reduz o ruído. Já o parâmetro 'Start Y' ajusta a caixa para que a medição esteja centralizada no espectro. O parâmetro 'Start $X$ ' define-se o início da escala que resulta no aumento de uma área limitada do espectro, e o parâmetro 'End X', define-se o fim da escala, resultando no aumento de uma área limitada do espectro.

No retângulo maior, tem-se o gráfico do espectro eletromagnético da luz incidente com as cores representando qual parte do espectro de luz visível o comprimento de onda representa. O ícone 'Reference' habilita a referência para as medições de absorção, ou seja, é utilizado para medir a curva de resposta dos filtros de cores e a absorção de várias substâncias. O ícone 'Dips' habilita a marcação dos valores mínimos do gráfico e, de modo análogo, o ícone 'Peaks' habilita a marcação dos valores máximos do gráfico. O ícone 'Colors', ativa as cores relativas do comprimento de onda. Com o parâmetro 'Filter' é possível aumentar ou diminuir os ruídos do gráfico, no intervalo de 50 a 100 haverá um alargamento das linhas e uma redução na resolução, e o parâmetro 'Speed' é a velocidade de resposta, para valores baixos os ruídos de curta duração são eliminados. O ícone 'Trim Scale' marca os comprimentos de onda mais bem definidos do seu espectro. 
Figura 8: Interface do software Theremino operando para a análise de um espectro eletromagnético.

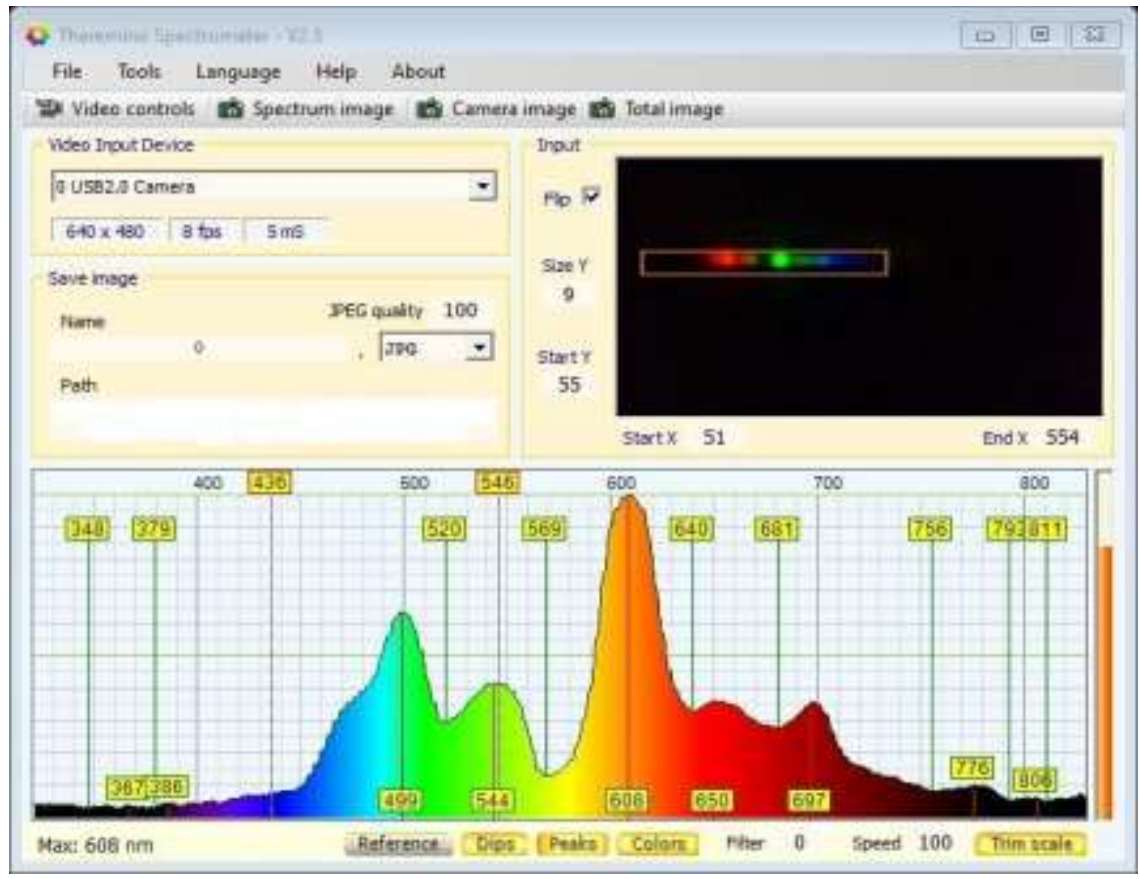

Fonte: Autores.

Utilizando a montagem do espectrômetro, juntamente com o software Theremino é possível visualizar na tela do computador os diferentes picos de radiação pertencentes a uma dada fonte do espectro. Por exemplo, é possível conseguir espectros por meio de um feixe de luz produzido em um tubo de descarga elétrica e elevadas temperaturas e baixas pressões (lâmpadas), que contém gases de determinados elementos como o hidrogênio, sódio, mercúrio ou berílio. Quando a luz passa pela lente da webcam é possível obter o espectro de emissão do elemento que compõe a lâmpada.

Um fator importante para a calibração do espectrômetro é fixar a câmera dentro da caixa a um ângulo de aproximadamente 30 graus. Tal escolha se justifica devido a posição da formação dos dois máximos de primeira ordem, como pode ser visto na Figura 9. Isso pode ser feito com o auxílio de um transferidor e ou por meio de marcações que podem ser realizadas no interior da caixa. A fenda de entrada da luz deve estar na horizontal e localizada aproximadamente na altura da ponta da lente. O ângulo de inclinação fornece um bom compromisso entre resolução e quantidade de luz coletada, mas é possível experimentar diferentes ângulos. Diminuindo o ângulo, a resolução aumenta, as linhas se movem para a esquerda e a intensidade da luz diminui, aumentando no caso contrário. Dependendo da sensibilidade da CCD, a distância focal de sua objetiva, seu número de pixels, o número de linhas na grade (CD, DVD ou rede 500 ou 1000 linhas) e quanta resolução você deseja, o ângulo ajustado pode diferir um pouco do valor de 30 graus indicados neste trabalho. 
Figura 9: Imagem do máximo de ordem zero (cor branca) e os máximos de ordem zero, obtidas no laboratório do Instituto de Física e Química utilizado uma fonte de luz branca e uma rede de difração com constante de rede $1,00 \times 10^{-6} \mathrm{~m}$.

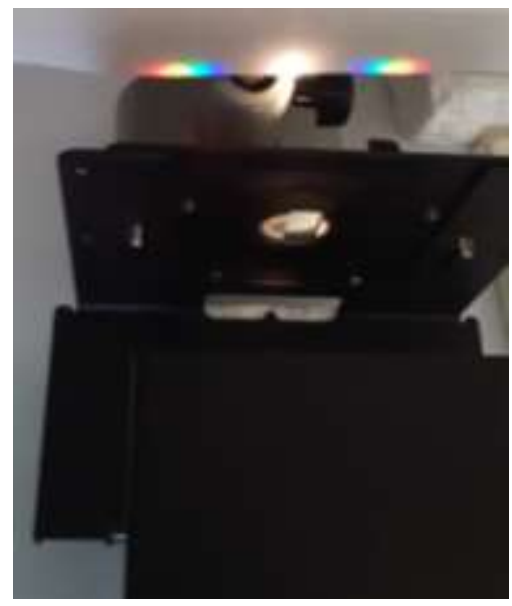

Fonte: Autores.

Na calibração foi utilizada uma lâmpada fluorescente. Essas lâmpadas geram duas linhas de mercúrio que são perfeitas para calibrar o espectrômetro. A lâmpada utilizada neste trabalho possui uma potência de $25 \mathrm{~W}$ e chega a uma temperatura de 6400 K. Na Figura 10 é apresentado o espectrômetro digital acondicionado na caixa e conectado ao software Theremino de visualização e armazenamento dos dados na etapa de calibração com a lâmpada fluorescente.

Figura 10: Espectrômetro digital acondicionado na caixa, conectado ao computador, em comunicação com o software de visualização e aquisição de dados Theremino.

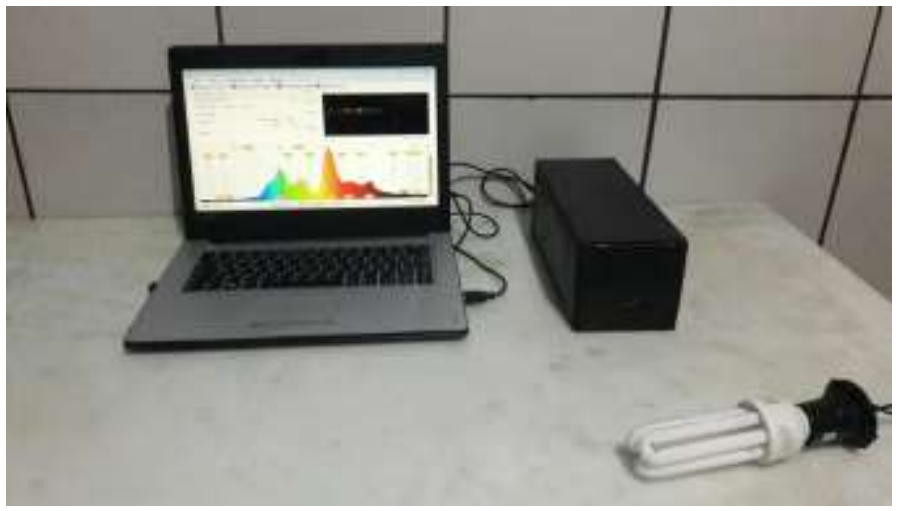

Fonte: Autores.

\section{Resultados e Discussão}

Após a etapa de construção do experimento foram selecionadas algumas fontes de radiação para testar o experimento, verificando sua viabilidade para utilização em aulas presenciais e remotas. Na Figura 11(a) é apresentado o espectro de uma lâmpada fluorescente de $25 \mathrm{~W}$ de potência. Este espectro permitiu identificar picos em uma ampla faixa do espectro eletromagnético. Além disso, na Figura 10(a) também foi possível verifica duas linhas escuras, entre 569 nm e 608 nm, o que significa que a lâmpada utilizada não possui nenhuma emissão nessas frequências. 
Figura 11: Espectro observado na lâmpada fluorescente de 25 W. (a) o espectro capturado pela webcam e (b) o gráfico apresentado pelo software Theremino.

(a)

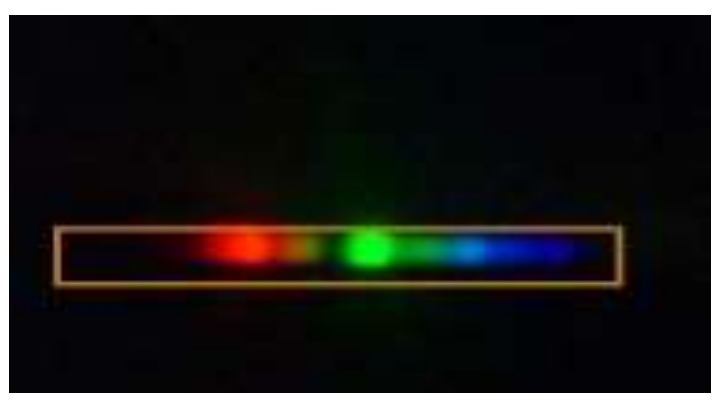

(b)

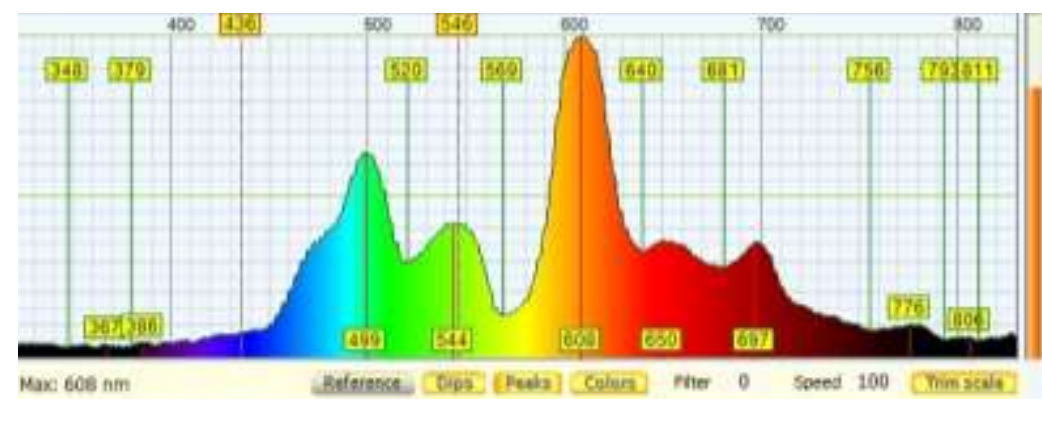

Fonte: Autores.

Na figura 11 (b) o espectro eletromagnético é apresentado de forma bem definida nos comprimentos de onda em 446 $\mathrm{nm}$ e $546 \mathrm{~nm}$, representando respectivamente as cores azul e verde, características típicas de uma lâmpada fluorescente que contém mercúrio. Desse modo foi possível fazer a calibração do software que já tem esses picos estabelecidos para a calibração do espectrômetro referente a uma lâmpada fluorescente.

Dando sequência aos testes do experimento, foi realizada uma medida com uma lâmpada incandescente. Como pode ser visto na Figura 12 (b), assim como previsto, o espectro da lâmpada incandescente tem o comportamento mais próximo contínuo, sem a existência de muitos picos que podem prejudicar o olho humano, como ocorre na lâmpada fluorescente (Rubinger, et al. 2015). A seguir foi utilizada uma lâmpada LED. No espectro apresentado na Figura 12 (c) podemos verificar a presença de três picos muito bem definidos nas cores vermelha, amarela e azul. Tal composição de cores gera a cor branca, que é interessante para iluminação de ambientes internos em diferentes ambientes. Seguindo nossa série de experimentos, que também são sugestões para o docente trabalhar com seus discentes em aulas presenciais e ou remotas, apresentamos nas Figuras 12(d), 12(e) e 12(f) os espectros da chama de uma vela, da luz solar e de um LED de controle remoto, respectivamente. Nesses espectros o discente pode diferenciar as intensidades em diferentes comprimentos de onda para diferentes fontes de radiação. 
Research, Society and Development, v. 10, n. 8, e51410817786, 2021

(CC BY 4.0) | ISSN 2525-3409 | DOI: http://dx.doi.org/10.33448/rsd-v10i8.17786

Figura 12: (a) Espectro da lâmpada fluorescente, (b) espectro da lâmpada incandescente, (c) espectro de uma lâmpada de LED, (d) espectro de uma vela, (e) espectro da luz solar e (f) espectro da radiação emitida por um controle remoto, obtidos com o espectrômetro digital construído neste trabalho em conjunto com o software Theremino.

(a)

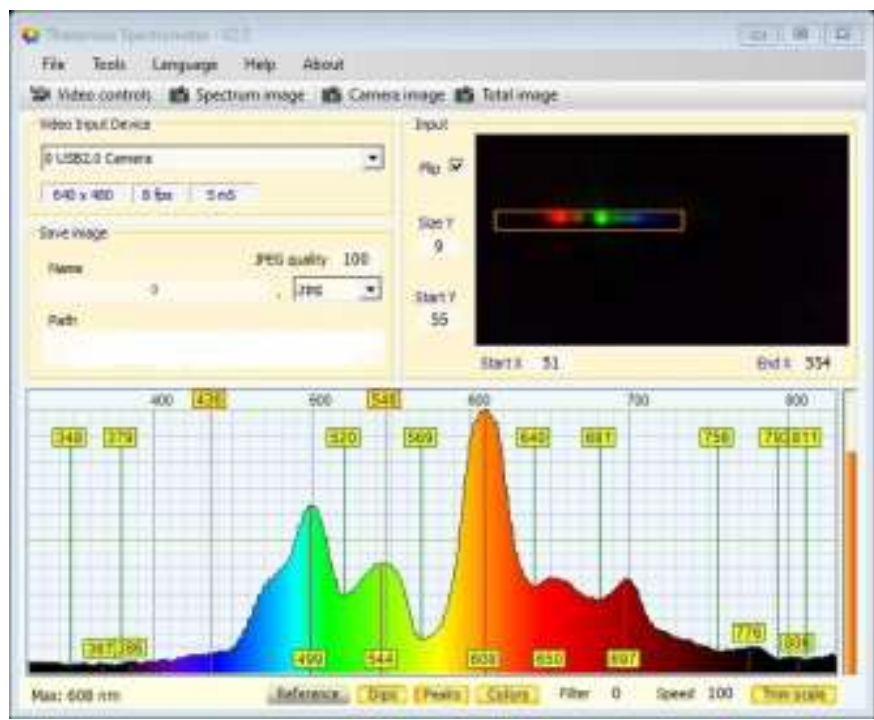

(c)

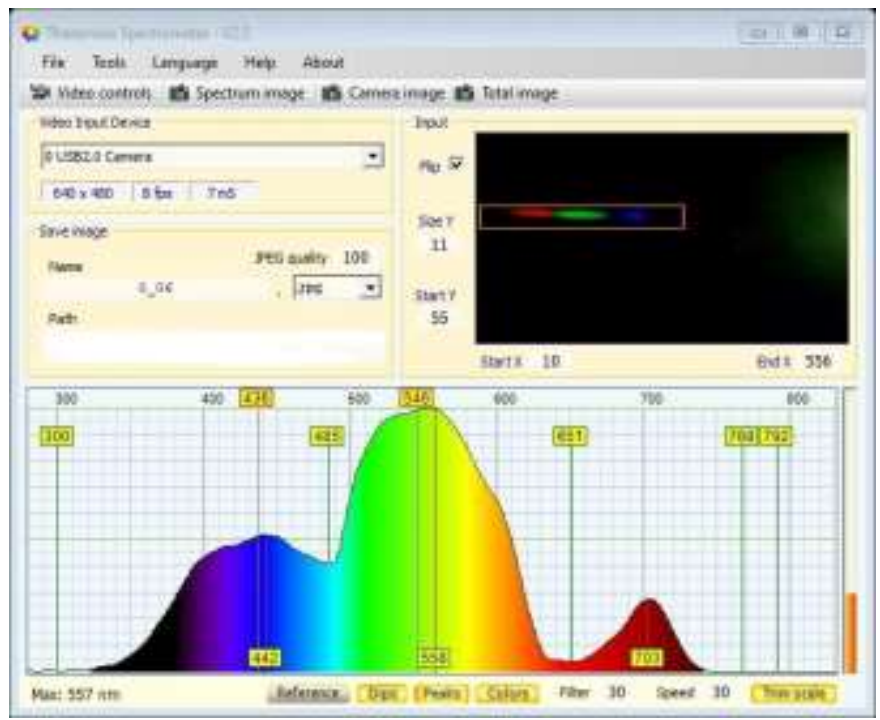

(e) (b)

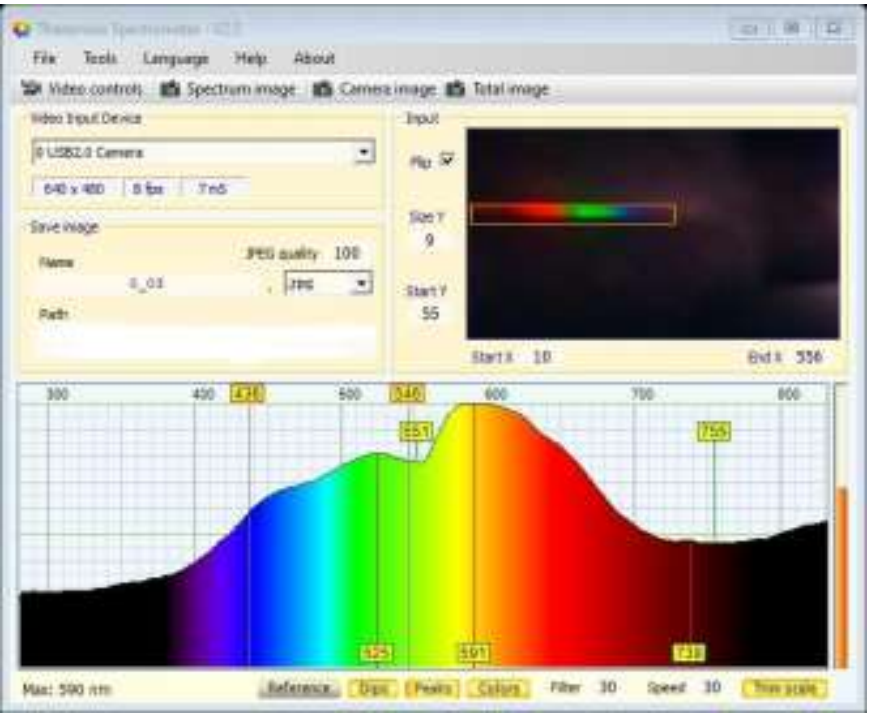

(d)

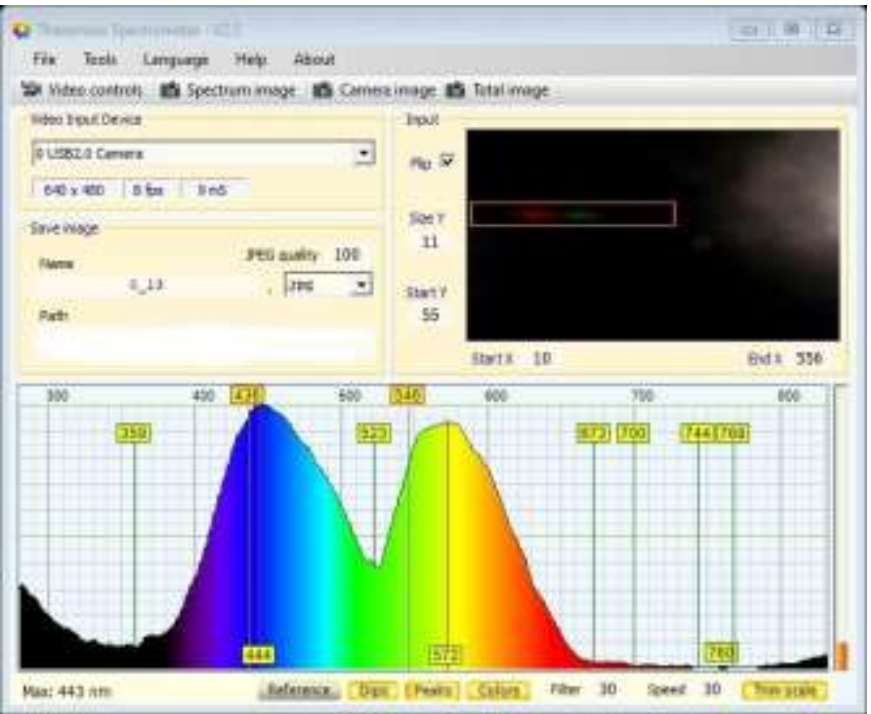

(f) 

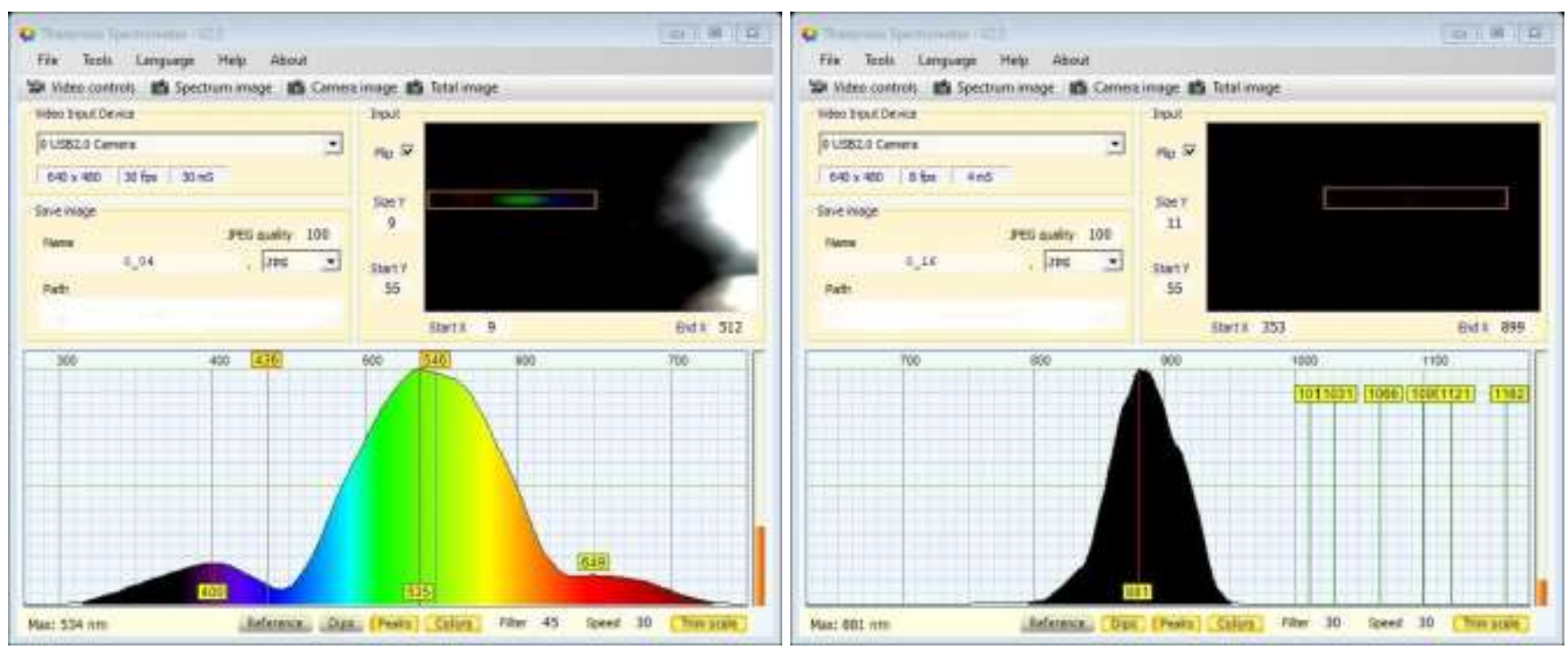

Fonte: Autores.

Além dos experimentos realizados com diferentes fontes de radiação, verificamos que o sistema funciona muito bem em paralelo com qualquer aplicativo de aulas online no formato síncrono (ex. Meet, Zoom, e outros). Para melhorar a atividade outra webcam pode ser utilizada para mostrar em tempo real a realização dos experimentos pelo docente. Esse experimento é muito importante para a formação do discente, pois além de apresentar informações presentes nos conceitos relacionados aos espectros de fontes emissoras de radiação eletromagnética e da difração da radiação, também introduz o discente a uma ferramenta que é utilizada na pesquisa em diversas áreas da ciência. Este experimento irá permitir ao docente introduzir conceitos e realizar diferentes medidas, tais como transmitância, refletância e absorbância da radiação através de filtros. Dentre muitos exemplos, podemos citar uma utilizando filtro solar depositado sobre uma superfície de uma lamina de vidro. Tal experimento irá permitir verificar a região do espectro que é absorvida para proteger a pele humana da radiação. Sendo esse, apenas um exemplo, das inúmeras atividades que podem ser desenvolvidas pelo docente juntamente com os discentes.

\section{Conclusão}

Neste trabalho é apresentada a construção de um espectrômetro digital, de baixo custo, que pode ser utilizado pelo docente em aulas presenciais e/ou remotas, principalmente neste período de pandemia, em que é necessário o distanciamento social. Após a montagem do experimento foram realizados diversos ensaios que mostraram a viabilidade de utilização do experimento e algumas informações que ele pode apresentar durante a realização da atividade experimental proposta. Na pesquisa científica o espectrômetro digital é muito utilizado, em diversos campos. Com isso, oferecemos aos docentes uma opção de um experimento que será fundamental para apresentar conceitos da interação do átomo com a radiação e outros temas relacionados a ciências e introduzir os discentes a pesquisa. Tal atividade pode tornar a formação significativa para os discentes, pois terão a oportunidade de enxergar a luz de uma forma diferente através da utilização dos espectrômetros.

Tendo em vista as inúmeras funcionalidades do software Theremino, nosso grupo pretende construir uma fonte de radiação que cubra o espectro eletromagnético entre os comprimentos de onda de 300nm e 900 nm para a realização de medidas de interferência em filmes finos. Tais medidas serão interessantes para estimar a espessura dos filmes (Stenzel, 2016) 


\section{Agradecimentos}

Os autores agradecem a FAPEMIG, Capes e CNPq pelo apoio financeiro. Além disso, agradecem ao Espaço InterCiências da Universidade Federal de Itajubá juntamente com o programa PET - Conexão de Saberes: Professores em Ciências Exatas.

\section{Referências}

Alves, V. C. \& Stackak, M. (2005) A importância de aulas experimentais no processo ensino aprendizagem em Física, XVI SNEF

Araújo, Mauro Sérgio Teixeira de, \& Abib, Maria Lúcia Vital dos Santos. (2003). Atividades experimentais no ensino de física: diferentes enfoques, diferentes finalidades. Revista Brasileira de Ensino de Física, 25(2), 176-194

Barros, L. G., Assis, A., \& Langhi, R. (2016). Proposta de construção de espectroscópio como alternativa para o ensino de Astronomia. Caderno Brasileiro de Ensino de Física, 33(3), 1026.

Barin, C., Saidelles, T., Schollmeier, A., Lampe, L., \& Ellensohn, R. (2021) Passeio virtual no laboratório de química: Uma alternativa para mediação pedagógica em tempos de Covid-19 ReTER, 2(2), e5/01-15.

Calheiro, L. B. \&; Del Pino, J. C. (2017) A compreensão dos conceitos de radiação eletromagnética no ensino médio à luz dos campos conceituais de Vergnaud, Enseñanza de las ciencias, Núm. Extra, p. 4395-4400

Catelli, F. (1999) projeção de espectros com um cd e retroprojetor, Caderno Brasileiro de Ensino de Física, Vol. 16, №. 1,123-126.

Catelli, F., \& Libardi, H. (2010). CDs como lentes difrativas. Revista Brasileira de Ensino de Física, 32(2).

dos Santos, C., da Silveira Freitas, P., \& Marchezan Lopes, M. (2020). Ensino Remoto e a utilização de laboratórios virtuais na área de ciências naturais, Anais Do Salão Internacional De Ensino, Pesquisa E Extensão, 12(1).

Eberhardt, D., Da Rocha Filho, J. B., Lahm, R. A., \& Baitelli, P. B. (2017). Experimentação no ensino de Física Moderna: efeito fotoelétrico com lâmpada néon e LEDs. Caderno Brasileiro de Ensino de Física, 34(3), 928-950.

Guenther, M. (2020). Como será o amanhã? O mundo pós-pandemia. Revista Brasileira de Educação Ambiental, 15(4), 31-44.

Kittel, C (2004) Introduction to Solid State Physics, John Wiley \& Sons; 8 a edição

Klaus, H. (1993) The Discovery of the Redshift of Solar Fraunhofer Lines by Rowland and Jewell in Baltimore around 1890. Historical Studies in the Physical and Biological Sciences, vol. 23, no. 2, pp. 219-277

Lima, N. W., Ostermann, F., \& De Holanda Cavalcanti, C. J. (2017). Física Quântica no ensino médio: uma análise bakhtiniana de enunciados em livros didáticos de Física aprovados no PNLDEM2015. Caderno Brasileiro de Ensino de Física, 34(2), 435.

Marinho, R.F., Vailant, C.C.R., Costaco, I.L.L. \& da Silva L.P.V. (2015) Medidas dos comprimentos de onda do espectro visível: Uma proposta didática de baixo custo, Anais da Semana de Licenciatura, 407.

Moraes, J. U. P \& Silva Jr., R. S. (2014) Experimentos didáticos no ensino de física com foco na aprendizagem significativa. Aprendizagem Significativa em Revista. 4(3), 61-67.

Oliveira, I. N., Ramos, J. A. P., Silva, W. L., Chaves, V. D., \& De Melo, C. A. O. (2020). Construção de uma maquete experimental automatizada para a determinação da constante de Planck com o auxílio da plataforma Arduíno. Caderno Brasileiro de Ensino de Física, 37(2), 828-848

Perkampusmar, H. H. (2013) UV-VIS Spectroscopy and Its Applications, Springer Science \& Business Media

Pereira, A.S., Shitsuka, D. M., Parreira, F. J., Shitsuka, R. (2018) Metodologia de pesquisa científica, UFSM

Schuchat, A. (2020). Public Health Response to the Initiation and Spread of Pandemic COVID-19 in the United States, February 24-April 21, 2020. MMWR. Morbidity and Mortality Weekly Report, 69(18), 551-556.

Silva, J. C. X., \& Leal, C. E. dos S. (2016). Proposta de laboratório de física de baixo custo para escolas da rede pública de ensino médio. Revista Brasileira de Ensino de Física, 39(1).

Silva, J. N. A., Neto, J. A. D. V., Ximenes, C. A. P., \& Morais, A. C. S. (2020). A experimentação como ferramenta motivacional no ensino de física / experimentation as a motivational method in physics teaching. Brazilian Journal of Development, 6(12), 102473-102485.

Stenzel, O. (2016). The Physics of Thin Film Optical Spectra. In Springer Series in Surface Sciences. Springer International Publishing.

Theremino, (2021). Educação e entretenimento. Website Theremino. https://www.theremino.com/pt/introduction/systems

Toledo, R. P., Huanca, D. R., Oliveira, A. F., dos Santos Filho, S. G., \& Salcedo, W. J. (2020). Electrical and optical characterizations of erbium doped MPS/PANI heterojunctions. Applied Surface Science, 529, 146994. 
Research, Society and Development, v. 10, n. 8, e51410817786, 2021

(CC BY 4.0) | ISSN 2525-3409 | DOI: http://dx.doi.org/10.33448/rsd-v10i8.17786

Silva, JCX, \& Leal, CE dos S. (2016). Proposta de laboratório de física de baixo custo para escolas de rede pública de ensino médio. Revista Brasileira de Ensino de Física, 39 (1).

Thomaz, M. F. (2000) A experimentação e a formação de professores de ciências: uma reflexão, Caderno Brasileiro de Ensino de Física, v.17, n.3: p.360-369

Rubinger, R. M., da Silva, E. R., Pinto, D. Z., Rubinger, C. P. L., Oliveira, A. F., \& da Costa Bortoni, E. (2015). Comparative and quantitative analysis of white light-emitting diodes and other lamps used for home illumination. Optical Engineering, 54(1), 014104. 